\title{
III. Ueber die electromagnetische Drehung der
} Polarisationsebene des Liahtes in Eisenchlorid; von Wladimir stscheglajeff aus Moskau. (Hierza Tap. II Fig. 20.)

Als Verdet gefunden hatte, dass concentrirte Lösungen von Eisenchlorid eine negative Drehung der Polarisationsebene des Lichtes im magnetischen Feld zeigen, und dass bei anderen Salzen magnetischer Metalle wenigstens das sogenannte ,moleculare Drehvermögen" negativ ist, lag es nahe, anzunehmen, dass die magnetischen Metalle für sich die $\mathrm{Po}$ larisationsebene in einem den Ampère'schen Molecularströmen entgegengesetzten, also negativen Sinn drehten, und dass dies negative Drehvermögen des Metalles selbst auch die negative Drehung der betreffenden Salze bedinge. Nachdem $\mathrm{Hr}$. $\mathrm{Kundt}$ gezeigt hat, dass die Metalle Eisen, Cobalt und Nickel die Polarisationsebene des hindurchgehenden Lichtes im positiven Sinn drehen, ist die obige Annahme hinfällig geworden. Damit hat aber die Thatsache, dass die Eisensalzlösungen und andere Metallsalzlösungen negative Drehung zeigen, nicht an Interesse verloren, vielmehr eher gewonnen. Will man eine Grundlage für die Erklärung des auffälligen Verhaltens der Lösungen stark magnetischer Salze erhalten, so wird es zunächst nöthig sein, die Erscheinung der electromagnetischen Drehung bei denselben möglichst nach allen Richtungen experimentell zu untersuchen. Bei diamagnetischen Substanzen ist, soweit bisher die Versuche reichen, die Drehung proportional der Intensität des magne. tischen Feldes; ausgedehntere Versuche darüber, ob dies auch bei den negativ drehenden Eisensalzlösungen der Fall sei, liegen bisher nicht vor.

Auf Veranlassung des Hrn. Professor Kundt habe ich es daher unternommen, zu untersuchen, ob und in welcher Weise sich die Drehung in Eisenchloridlösungen mit der Intensität des Magnetfeldes ändere. Die folgende Mittheilung enthält die Resultate dieser Untersuchung. Ich gebe in $\$ 1$ eine Beschreibung der Versuchsanordnung, in $\S 2$ die Beobachtungen, in $\S 3$ eine kurze Discussion derselben. 
\$1. Beschreibung der Apparate und der Untersuchungsmethode.

Ich benutzte bei meinen Untersuchungen einen grossen Electromagnet, dessen Eisenkern aus zwei massiven Cylindern, $47 \mathrm{~cm}$ hoch und $11 \mathrm{~cm}$ im Durchmesser, besteht. Auf jedem Cylinder befinden sich je drei Rollen von $3 \mathrm{~mm}$ dickem Kupferdraht. Der Widerstand einer jeden Rolle ist ungefähr gleich $0,65 \mathrm{~S}$.-E. Die Cylinder tragen massive eiserne Anker, welche durch einen runden Canal von ungefähr $25 \mathrm{~mm}$ Durchmesser durchbohrt sind. In den Canal sind eiserne Röhren eingeschoben, die an ihren Enden kegelförmige Polstücke mit Oeffnungen von $5 \mathrm{~mm}$ Durchmesser tragen. Damit die Pole unter Wirkung der magnetischen Anziehung nicht näher aneinander rücken könnten, waren die oberen Enden der Cylinder durch ein festes hölzernes Brett getrennt, dessen abgerundete Ränder genau an die $\mathrm{Cy}$ linder angepasst waren. Dieses Brett diente gleichzeitig zum Aufstellen der zu untersuchenden Stoffe. Den erregenden electrischen Strom lieferte eine Batterie oder eine Gramme'sche Maschine. Die Batterie bestand aus grossen Bunsen'schen Elementen. Indem die Zahl der letzteren von 4 bis 12 geändert wurde, konnte die Stromstärke von 2,3 bis 7 Ampère erhöht werden. Der Maschinenstrom wurde vermittelst eines in die Kette eingeführten Widerstandes von 9,7 bis 13,9 Ampere rerändert. Die Constanz des Stromes konnte durch ein eingeschaltetes Deprez'sches Ampèrometer controlirt werden. Ein Quecksilbercommutator erlaubte, die Richtung des Stromes im Electromagnet umzukehren. Das Feld zwischen den conischen Polen ist in grösserer Ausdehnung selbstverständlich nicht homogen; es wurde deshalb Sorge getragen, dass die Substanzen, deren Drehung bestimmt werden sollte, stets genau an dieselbe Stelle zwischen die Pole gebracht wurden. Die Flussigkeiten befanden sich in zwei genau gleichen Gefässen mit planparallelen Wänden. Diese Gefässe bestanden aus zwei passend ausgeschnittenen Stücken einer und derselben Spiegelglasplatte, auf deren Oefinungen vermittelst Canadabalsam dünne Deckgläschen, wie sie für mikroskopische $\mathrm{Z}_{\text {wecke }}$ benutzt werden, aufgeklebt waren. 
Die untersuchten Eisenchloridlösungen, die im Folgenden mit $1,2,3,4$ bezeichnet sind, hatten bei mittlerer Temperatur die Dichten:

$$
1,463, \quad 1,338, \quad 1,239, \quad 1,109 .
$$

Als Lichtquelle diente ein Argand'scher Brenner, dessen: Strahlen durch ein rothes Glas gingen. Der Polarisator war ein Jellet'sches Prisma. Das analysirende Nicol'sche Prisma befand sich in einem Theilkreis, dessen Nonius einen. Winkel von vier Minuten unmittelbar ablesen liess und folg. lich eine Genauigkeit von zwei Minuten erlaubte. Der Unterschied zwischen den äussersten Werthen in einem Satz von zehn Ablesungen erreichte nur sehr selten 10 .

Die Drehungen wurden folgenderweise gemessen. Nach Einstellung der untersuchten Substanz im Magnetfelde wurde der Strom geschlossen und zehn Einstellungen des Analysators gemacht. Dann wurde der Strom in den Rollen des. Electromagnets commutirt und wiederum zehn Einstellungen bei der neuen Stromrichtung gemacht u. s. w. Zwei solche Sätze bei der einen und der dazwischenliegende bei der anderen Stromrichtung wurden zu einem Gesammtresultate zusammengefasst, sodass für jedes derartige Resultat 30 Einstellungen erforderlich waren. Die Drehungen der Polarisationsebene des Lichtes im Eisenchlorid wurden mit denen im Wasser und im Glase verglichen.

Die Stromstärke der Batterie sinkt mit der Zeit; um nun alle Drehungen bei derselben Stärke des Stroms und folglich des Magnetfeldes vergleichen zu können, wurden die Messungen nach dem folgenden Programm ausgeführt: Drehung durch 1. Eisenchlorid, 2. Wasser, 3. Glas, 4. Eisenchlorid, 5. Wasser, 6. Glas u. s. w.

Das Mittel von den Messungen (1) und (4) für Eisenchlorid wurde mit der Messung (2) für Wasser oder (3) für Glas ver. glichen; umgekehrt wird das Mittel von (3) und (6) für Glas oder von (2) und (5) für Wasser als Maass der Intensität des Magnetfeldes während der Messung der Drehungen im Eisenchlorid (4) betrachtet. Bei der Untersuchung der Lö. 
sungen Nr. 3 und Nr. 4, welche weniger licht absorbiren und folglich den Analysator genauer einzustellen erlauben, war das oben erwähnte Programm verkürzt. Hier wurde nämlich zwischen zwei Messungen der Drehungen im Wasser und im Glase die Drehung im Eisenchlorid nur einmal gemessen, es wurde aber dabei die Stromrichtung fünfmal commutirt, woraus fünf Einstellungssätze entstanden. Die Combination dreier benachbarter Sätze gab die Grösse der Doppeldrehung im Eisenchlorid, und die drei auf diese Weise erhaltenen Zahlen wurden mit dem Mittelwerth der Drehung im Glase oder im Wasser verglichen. Um die Drehung im Wasser mit der im Glase zu vergleichen, wurden die Zahlen auf dieselbe Weise combinirt. Die Drehungen im Wasser und im Eisenchlorid wurden in Bezug auf die Drehungen in den Wänden der sie enthaltenden Gefässe corrigirt. $\mathrm{Zu}$ diesem $\mathrm{Z}$ wecke wurden jedesmal die Drehungen in den Wänden der leeren Gefässe aus mehreren Einstellungen bestimmt. Die Grösse der doppelten Drehung in den Wänden der Gefässe schwankte von $+6^{\prime}, 4$ bis $+13^{\prime}, 6$; diese Grösse wurde von den beobachteten Grössen der doppelten Drehungen im Wasser abgezogen und zu den negativen doppelten Drehungen im Eisenchlorid addirt.

\section{\$ 2. Die Beobachtungen.}

Die erhaltenen Resultate sind in den folgenden Tabellen wiedergegeben, worin $E, G, W$ die doppelten Drehungen im Eisenchlorid, Glas und Wasser und $R_{e g}$ und $R_{g w}$ die Verhältnisse $E / G$ und $G / W$ bezeichnen. Sämmtliche Beobachtungen sind mit rothem Licht angestellt; die Dicke der Flüssigkeitsgefässe betrug $3,638 \mathrm{~mm}$, die des Glases $3,692 \mathrm{~mm}$.

Tabelle I. Eisenchloridlösung Nr. 1.

\begin{tabular}{c|c|c|c|c|c|c}
\hline $\begin{array}{c}\text { Stromstärke } \\
\text { in Ampere }\end{array}$ & $-E$ & $G$ & & $W$ & $-R_{e g}$ & $R_{g w}$ \\
\hline \begin{tabular}{c|c}
2,35 \\
(4 Bunsen)
\end{tabular} & $5^{0} 39,3^{\prime}$ & $1^{0} 24,4^{\prime}$ & $1^{0}$ & $7,0^{\prime}$ & 4,020 & 1,259 \\
\hline Mittel & $5^{0} 38,75^{\prime}$ & $1^{0} 23,75^{\prime}$ & $1^{0}$ & 6,8 & 4,045 & 1,260
\end{tabular}


(Fortsetzung der Tabelle I.)

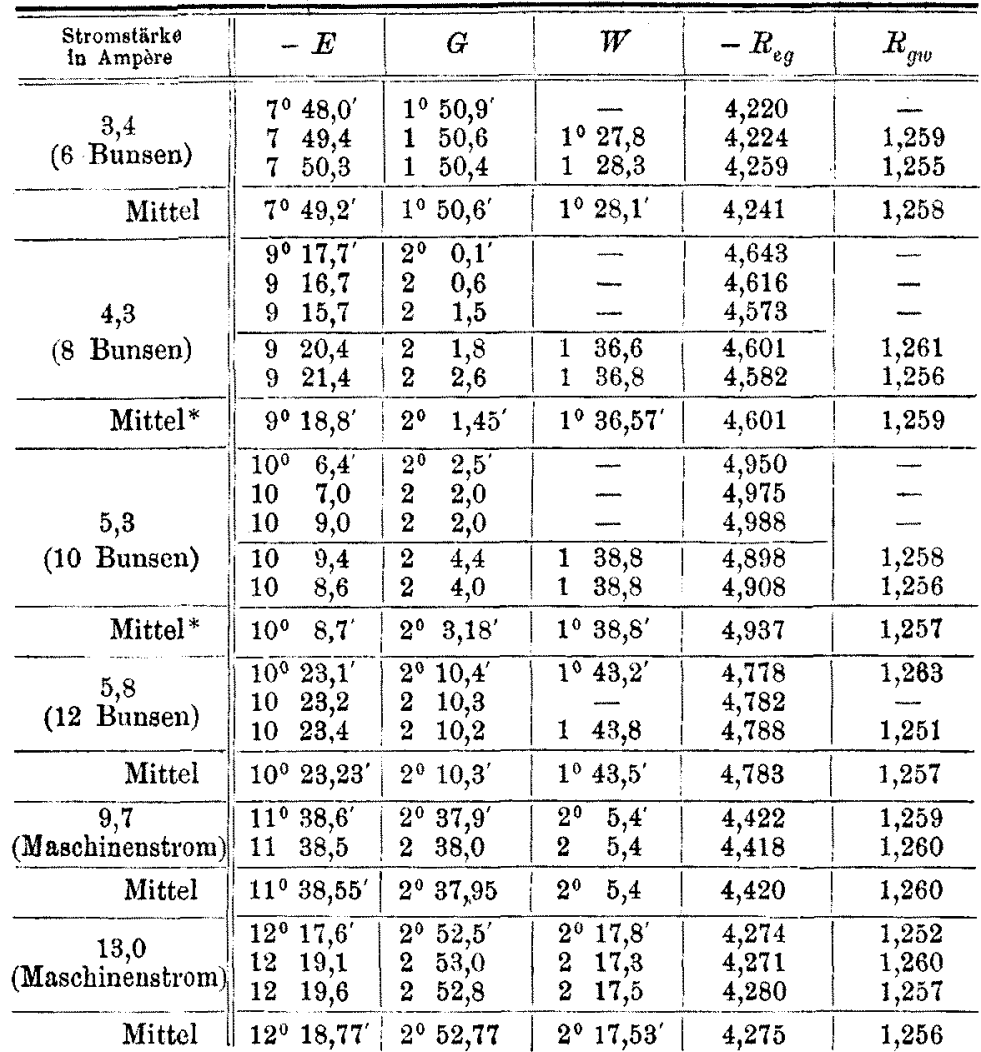

* Das Mittel ist gewonnen, indem die drei ersten und die zwei letzten Beobachtungen zusammengefasst wurden.

Tabelle II. Eisenchloridlösung Nr. 2.

\begin{tabular}{|c|c|c|c|c|c|}
\hline $\begin{array}{c}\text { Stromatärire } \\
\text { in Amperre }\end{array}$ & $-E$ & $G$ & $W$ & $-R_{e g}$ & $R_{g w}$ \\
\hline (4 Bunsen) & $\begin{array}{l}2^{\circ} 36,4^{\prime} \\
2 \quad 36,1 \\
\end{array}$ & $\begin{array}{l}1^{0} 21,6^{\prime} \\
1 \quad 21,4\end{array}$ & $\begin{array}{ll}1^{0} & 4,6 \\
1 & 4,8 \\
\end{array}$ & $\begin{array}{l}1,917 \\
1,918\end{array}$ & $\begin{array}{l}1,263 \\
1,256\end{array}$ \\
\hline Mittel & $2^{0} 36,25^{\prime}$ & $1^{0} 21,5^{\prime}$ & $1^{0} 4,7^{\prime}$ & 1,917 & 1,260 \\
\hline (6 Bunsen) & $\begin{array}{l}3^{0} \quad 44,0^{\prime} \\
8 \quad 43,7 \\
\end{array}$ & $\begin{array}{l}1^{0} 49,9^{\prime} \\
1 \quad 50,6 \\
\end{array}$ & $\begin{array}{l}1^{0} 27,4^{\prime} \\
127,3\end{array}$ & $\begin{array}{l}2,038 \\
2,022 \\
\end{array}$ & $\begin{array}{l}1,257 \\
1,266 \\
\end{array}$ \\
\hline Mittel & $3^{0} 43,85^{\prime}$ & $1^{0} 50,25^{\prime}$ & $1^{0} 27,35^{\circ}$ & 2,030 & 1,262 \\
\hline
\end{tabular}


(Fortsetzung von Tabelle Il.)

\begin{tabular}{|c|c|c|c|c|c|}
\hline $\begin{array}{c}\text { Stromstärike } \\
\text { In Ampere }\end{array}$ & $-E$ & $G$ & $W$ & $-R_{e g}$ & $R_{g w}$ \\
\hline$\left(\begin{array}{c}5,0 \\
(8 \text { Bunsen) }\end{array}\right.$ & $\begin{array}{l}4^{0} \quad 29,0^{\prime} \\
4 \quad 28,4 \\
\end{array}$ & $\begin{array}{ll}2^{0} & 4,8^{\prime} \\
2 & 4,4 \\
\end{array}$ & $\begin{array}{l}1^{0} 39,0^{\prime} \\
1 \quad 39,1 \\
\end{array}$ & $\begin{array}{l}2,156 \\
2,157\end{array}$ & $\begin{array}{l}1,261 \\
1,255\end{array}$ \\
\hline Mittel & $4^{0} 28,7^{\prime}$ & $2^{0} \quad 4,6^{\prime}$ & $1^{0} 39,05^{\prime}$ & 2,157 & 1,258 \\
\hline $\begin{array}{c}6,0 \\
(10 \text { Bunsen })\end{array}$ & $\begin{array}{l}4^{0} 38,4^{t} \\
4 \quad 38,3\end{array}$ & $\begin{array}{ll}2^{0} & 13,5^{\prime} \\
2 & 14,0 \\
\end{array}$ & $\begin{array}{l}1^{0} 46,8^{\prime} \\
1 \quad 46,4\end{array}$ & $\begin{array}{l}2,085 \\
2,076\end{array}$ & $\begin{array}{l}1,250 \\
1,259\end{array}$ \\
\hline Mittel & $4^{0} 38,35^{\prime}$ & $2^{0} 13,75^{\prime}$ & $1^{0} 46,6^{\prime}$ & 2,081 & 1,255 \\
\hline (12 Bunsen) & $\begin{array}{l}4^{0} 45,6^{\prime} \\
4 \quad 45,1 \\
\end{array}$ & $\begin{array}{l}2^{0} 21,6^{\prime} \\
2 \quad 22,7 \\
\end{array}$ & $\begin{array}{l}1^{0} \quad 53,2 \\
1^{2} 53,4 \\
\end{array}$ & $\begin{array}{l}2,016 \\
2,000\end{array}$ & $\begin{array}{l}1,251 \\
1,259\end{array}$ \\
\hline Mittel & $4^{0} 45,35^{\prime}$ & $2^{0} 22,15^{\prime}$ & $1^{0} 53,3^{\prime}$ & 2,008 & 1,250 \\
\hline $\begin{array}{c}13,9 \\
\text { (Maschinenstrom) }\end{array}$ & $\begin{array}{l}5^{0} 37,4^{\prime} \\
5 \quad 34,9 \\
\end{array}$ & $\begin{array}{ll}2^{0} & 58,7^{1} \\
2 & 57,4 \\
\end{array}$ & $\begin{array}{c}2^{0} 21,2^{\prime} \\
-\end{array}$ & $\begin{array}{l}1,888 \\
1,887\end{array}$ & 1,258 \\
\hline Mittel & $5^{0} 36,65^{\circ}$ & $2^{\circ} 58,05^{\prime}$ & $2^{0} 21,2^{\prime}$ & 1,888 & 1,258 \\
\hline
\end{tabular}

Tabelle III. Eisenchloridlösung Nr. 3.

\begin{tabular}{|c|c|c|c|c|c|}
\hline Stromstärke & $-E$ & $G$ & $W$ & $-R_{e g}{ }^{*}$ & $R_{g w}{ }^{*}$ \\
\hline 4 Bumsen & $\begin{array}{l}59,9^{\prime} \\
58,9\end{array}$ & $1^{0} 22,7^{\prime}$ & $\begin{array}{ll}1^{0} & 5,9^{\prime} \\
1 & 5,5 \\
\end{array}$ & $\begin{array}{l}0,724 \\
0,712\end{array}$ & $\begin{array}{l}1,255 \\
1,261\end{array}$ \\
\hline Mittel & $59,4^{\prime}$ & $1^{0} 22,7^{\prime}$ & $1^{0} \quad 5,7^{\prime}$ & 0,718 & 1,258 \\
\hline 8 Bunsen & $\begin{array}{ll}1^{0} & 47,5^{\prime} \\
1 & 47,2 \\
1 & 48,4\end{array}$ & $\begin{array}{ll} & - \\
2^{0} & 4,1^{\prime} \\
2 & 4,4 \\
\end{array}$ & $\begin{array}{l} \\
1^{\circ} \overline{38,5} \\
1 \quad 39,0 \\
\end{array}$ & $\begin{array}{l}0,861 \\
0,864 \\
0,874 \\
\end{array}$ & $\begin{array}{l}\overline{1,260} \\
1,257\end{array}$ \\
\hline Mittel & $1^{\circ} 47,5^{\prime}$ & $2^{0} \quad 4,3^{\prime}$ & $1^{0} 38,7^{\prime}$ & 0,866 & 1,258 \\
\hline 12 Bunsen & $\begin{array}{l}1^{\circ} 49,3^{\prime} \\
1 \quad 49,0 \\
\end{array}$ & $\begin{array}{lr}2^{0} & 15,9^{\prime} \\
2 & 15,6\end{array}$ & $\begin{array}{lr}1^{0} & 47,8^{\prime} \\
1 & 48,3\end{array}$ & $\begin{array}{l}0,804 \\
0,802\end{array}$ & $\begin{array}{l}1,261 \\
1,252\end{array}$ \\
\hline Mittel & $1^{0} 49,15^{\prime}$ & $2^{\circ} \quad 15,9^{\prime}$ & $1^{0} 48,1^{\prime}$ & 0,803 & 1,256 \\
\hline 16 Bunsen & \begin{tabular}{lr|}
$1^{0}$ & $53,7^{\prime}$ \\
1 & 52,6 \\
1 & 52,3
\end{tabular} & $\begin{array}{cc}2^{0} & 31,5^{\prime} \\
& - \\
2 & 30,7\end{array}$ & $\begin{array}{c}2^{0} \quad 0,3^{\prime} \\
-\overline{5} \\
1 \quad 5,9\end{array}$ & $\begin{array}{l}0,750 \\
0,743 \\
0,741\end{array}$ & $\begin{array}{c}1,259 \\
\overline{1,256}\end{array}$ \\
\hline Mittel & $1^{0} 52,9^{\prime}$ & $2^{0} 31,1^{\prime}$ & $2^{0} \quad 0,1^{\prime}$ & 0,745 & 1,258 \\
\hline
\end{tabular}

Tabelle IV. Eisenchloridlösung Nr. 4.

\begin{tabular}{|c|c|c|c|c|c|}
\hline Stromstärke & $-E$ & $G$ & $W$ & $-R_{e g}{ }^{*}$ & $R_{g w}{ }^{*}$ \\
\hline 4 Bunsen & $\begin{array}{l}41,6^{\prime} \\
42,2\end{array}$ & $1^{\circ} 22,2^{\prime}$ & $1^{0} 5,2^{\circ}$ & $\begin{array}{l}0,506 \\
0,513\end{array}$ & $\begin{array}{c}1,261 \\
-\end{array}$ \\
\hline Mittel & $41,9^{\prime}$ & $1^{0} 22,2^{\prime}$ & $1^{0} \quad 5,2$ & 0,510 & 1,261 \\
\hline
\end{tabular}

* Für die Berechnung der $R$ in III und IV vgl. p. 171. 
(Fortsetzung der Tabelle IV.)

\begin{tabular}{|c|c|c|c|c|c|}
\hline Stromstärke & $-E$ & $G$ & $W$ & $-R_{e g}{ }^{*}$ & $R_{g w}{ }^{*}$ \\
\hline 8 Bunsen & $\begin{array}{l}55,0^{\prime} \\
54,3 \\
54,0\end{array}$ & $\begin{array}{c}2^{0} \quad 3,9^{\prime} \\
-\end{array}$ & $\begin{array}{c}1^{0} 38,5 \\
-\end{array}$ & $\begin{array}{l}0,444 \\
0,438 \\
0,436\end{array}$ & $\begin{array}{c}1,255 \\
- \\
-\end{array}$ \\
\hline Mittel & 54,4 & $2^{0} \quad 3,9^{\prime}$ & $1^{\circ} 38,5^{\prime}$ & 0,439 & 1,255 \\
\hline 12 Bunsen & $\begin{array}{l}58,3^{\prime} \\
57,1 \\
57,9\end{array}$ & $\begin{array}{c}2^{0} 13,5 \\
2 \overline{12,8} \\
\end{array}$ & $\begin{array}{c}1^{0} 45,8^{\prime} \\
- \\
1 \quad 45,8\end{array}$ & $\begin{array}{l}0,438 \\
0,429 \\
0,435\end{array}$ & $\begin{array}{l}1,259 \\
1,250\end{array}$ \\
\hline Mittel & $57,8^{\prime}$ & $2^{0} 13,1^{\prime}$ & $1^{0} 45,8^{\prime}$ & 0,434 & 1,257 \\
\hline 16 Bunsen & $\begin{array}{ll}1^{0} & 1,3^{\prime} \\
1 & 1,7 \\
1 & 1,4\end{array}$ & $\begin{array}{c}2^{0} 29,7^{\prime} \\
2 \overline{30,2}\end{array}$ & $\begin{array}{c}1^{0} 58,5^{\prime} \\
-\overline{5} \\
158,8\end{array}$ & $\begin{array}{l}0, \overline{409} \\
0,412 \\
0,410\end{array}$ & $\begin{array}{c}1,262 \\
\overline{1,264}\end{array}$ \\
\hline Mittel & $1^{0} \quad 1,5^{\prime}$ & $2^{0} 29,9^{\prime}$ & $1^{0} 58,6^{\prime}$ & 0,411 & 1,263 \\
\hline
\end{tabular}

* Für die Berechnung der $R$ vgl. p. 171.

In den nachfolgenden Tabellen sind zur Uebersicht die Mittelwerthe der obigen Versuche noch einmal zusammen. gestellt.

Tabelle V. Eisenchloridlösung Nr. 1.



Tabelle VI. Eisenchloridlösung Nr. 2.

\begin{tabular}{|c|c|c|c|c|c|c|c|}
\hline \multicolumn{2}{|c|}{$E$} & \multicolumn{2}{|r|}{$G$} & \multicolumn{2}{|r|}{$W$} & $R_{e g}$ & $R_{g w}$ \\
\hline$-2^{0}$ & $36,25^{\prime}$ & $1^{0}$ & $21,5^{\prime}$ & & $4,7^{\prime}$ & $-1,917$ & 1,260 \\
\hline-3 & 43,85 & 1 & 50,2 & & 27,35 & $-2,030$ & 1,262 \\
\hline-4 & 28,7 & 2 & 4,6 & & 39,05 & $-2,157$ & 1,258 \\
\hline-4 & 38,35 & 2 & 13,75 & & 46,6 & $-2,081$ & 1,255 \\
\hline-4 & 45,35 & 2 & 22,25 & & 53,3 & $-2,008$ & 1,255 \\
\hline-5 & 36,65 & 2 & 58,05 & & 21,2 & $-1,888$ & 1,258 \\
\hline & & & & & & $\mathrm{Mit}$ & 1,2577 \\
\hline
\end{tabular}


Magnetisches Verhalten von Eisenchlorid.

Tabelle VII. Eisenohloridlösung Nr. 3.

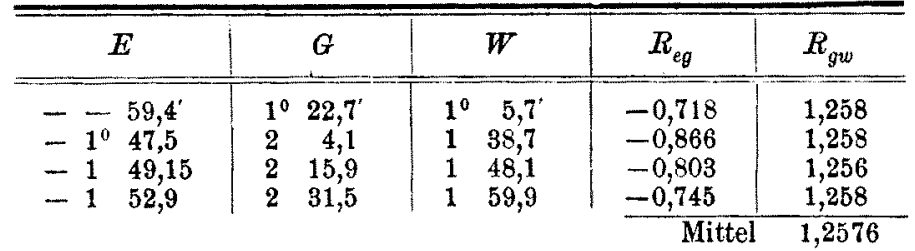

Tabelle VIII. Eisenchlorid. ösung Nr. 4.

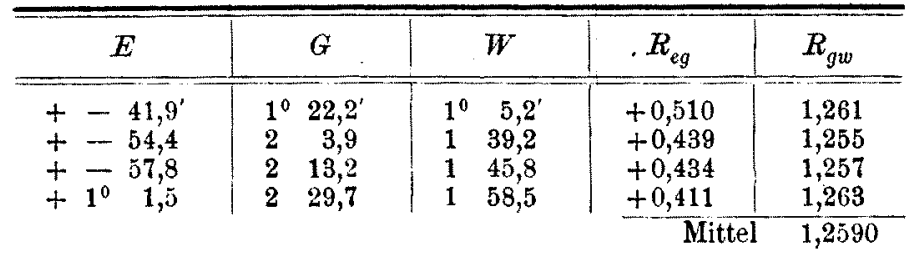

83. Discussion der Tabellen und Umrechnung auf absolutes Maass.

Aus den vorstehenden Tabellen ergibt sich zunächst, dass das Verhältniss der Drehungen im Wasser und Glas inner. halb der Beobachtungsfehler bei allen Intensitäten des Feldes constant bleibt. Es wird dadurch in hohem Grade wahrscheinlich, dass die Drehung sowohl in dem benutzten Glas wie in Wasser der Intensität des Feldes selbst proportional ist. Mit Hülfe der sogenannten Verdet'schen Constante für Wasser können wir mithin aus den beim Wasser beobachteten Drehungen die Intensität des Magnetfeldes in jedem einzelnen Fall in absolutem Maasse berechnen. Nach der Bestimmung des Hrn. Arons ${ }^{1}$ ) ist die Verdet'sche Constante für Wasser und Natronlicht bei. $23^{0} \mathrm{C} .0,3767.10^{-5} \mathrm{~cm}^{-1 / 2} \mathrm{~g}^{-1 / 2} \mathrm{sec} .1$, oder die Drehung in 1 Centimeter Wasser im Feld von der Intensität Eins, wenn die Lichtstrablen und die magnetischen Kraftlinien dieselbe Richtung haben, in Winkelmaass gleich 0,01295 .

Die Temperatur bei unseren Beobachtungen war im Mittel $20^{\circ} \mathrm{C}$., und es wurde, wie oben gesagt, Licht benutzt, welches durch ein rothes Glas gegangen war. Die Wellenlänge dieses Lichtes ist im Mittel gleich derjenigen der

1) Arons, Wied. Ann. 24. p. 180. 1885. 
Fraunhofer'schen Linie $C$ zu setzen. Nach H. Becquer el ${ }^{1}$ ) ist das Verhältniss der Drehung fur die Linie $C$ und $D$ im Wasser gleich 0,77. Reducirt man hiernach auf Licht der C-Linie und nach den Bestimmungen Bichat's ${ }^{2}$ ) über die Veränderung der Drehung des Wassers mit der Temperatur von $23^{\circ}$ auf $20^{\circ}$, so ergibt sich die Verd et'sche Constante für Wasser bei $20^{\circ}$ und Licht der $C$-Linie $w=0,2919.10^{-5} \mathrm{~cm}^{-1 / 2} \mathrm{~g}^{1 / 2} \mathrm{sec}^{1}$ oder in Winkelmaass gleich $0,01003^{\prime}$.

Die in den Versuchen benutzte Wasserschicht ebenso wie die Eisenchloridschicht hatte eine Dicke von $0,3638 \mathrm{~cm}$, mithin entspricht einem Grad doppelter Drehung in dieser Wasserschicht eine Intensität des Feldes von: $8221 \mathrm{~cm}^{-1 / 2} \mathrm{~g}^{1 / 2} \mathrm{sec}^{-1}$.

Mit Hülfe dieser Zahl sind für die Tabellen V, VI, VII, VIII aus den Drehungen im Wasser die Intensitäten des magnetischen Feldes berechnet, und aus den bei einer Schichtendicke von $0,3638 \mathrm{~cm}$ beobachteten Drehungen im Eisenchlorid ist jedesmal die Drehung für die Schicht $1 \mathrm{~cm}$ berechnet.

Die nachfolgenden Tabellen enthalten die berechneten Werthe, und zwar bezeichnet $H$ die Intensität des Feldes in c.g.s., $\Delta$ die einfache Drehung in Eisenchlorid bei der Schichtendicke $1 \mathrm{~cm}$ in dem betreffenden Feld, und zwar in Bogenmaass ausgedrückt. Die dritte Columne enthält die Quotienten $\Delta / H=\Omega$.

Wäre die Drehung im Eisenchlorid der Intensität des Feldes proportional, so müsste dieser Quotient constant sein, und der Werth desselben wäre die Verdet'sche Constante der betreffenden Eisenchloridlösung.

Den Tabellen IX bis XII entsprechen die beigegebenen Curven $A B, C D, E F, G H$. Als Abscissen sind die $H$, als Ordinaten die $\triangle$ aufgetragen.

Als wesentliches Resultat ergibt ein Blick auf die Curven:

1. Für Eisenchloridlösungen gilt das Verdet'sche Gesetz von der Proportionalität zwischen der electromagnetischen Drehung und der Intensität des Magnetfeldes nicht.

1) H. Becquerel, Wied. Electr. 3. p. 935 .

2) Bichat, 1. c. 3. p. 937. 
2. Die Drehung steigt bei den concentrirten Lösungen 1, 2, 3 anfangs schneller an, als der Proportionalität entspricht, während bei höheren Intensitäten das Verhältniss $\Delta / H$ wieder abnimmt.

Bei der Lösung schwächster Concentration, 4, ist. nur eine Abnahme von $\Delta / H$ erkennbar.

Tabelle IX.

Eisenchloridlösung Nr. 1.*

\begin{tabular}{r|c|c}
\hline \multicolumn{1}{c|}{$H$} & $-100 \mathrm{~A}$ & $-10^{7} \Omega$ \\
\hline \hline 9149 & 13,54 & 148,6 \\
$(9276$ & 13,77 & $148,4)$ \\
$(11856$ & 18,42 & $155,3)$ \\
12066 & 18,76 & 155,7 \\
13227 & 22,34 & 169,0 \\
$(13348$ & 23,82 & $178,1)$ \\
13532 & 24,32 & 180,9 \\
14176 & 24,92 & 175,6 \\
$(14578$ & 25,28 & $173,4)$ \\
17175 & 27,93 & 162,3 \\
18521 & 29,54 & 157,0
\end{tabular}

Tabelle XI.

Eisenchloridlösung Nr. 3.

\begin{tabular}{c|r|r}
\hline \multicolumn{1}{c}{$H$} & $-100 A$ & $-10^{7} \Omega$ \\
\hline 8998 & 2,374 & 26,37 \\
13519 & 4,298 & 31,81 \\
14806 & 4,378 & 29,49 \\
16422 & 4,513 & 27,36
\end{tabular}

Tabelle X.

Eisenchloridlösung Nr. 2.

\begin{tabular}{c|r|r}
\hline \multicolumn{1}{c|}{$H$} & $-100 \mathrm{~A}$ & $-10^{7} \Omega$ \\
\hline 8861 & $\mathbf{6 , 2 5}$ & $\mathbf{7 0 , 4 0}$ \\
11964 & 8,95 & $\mathbf{7 4 , 5 5}$ \\
13577 & 10,74 & $\mathbf{7 9 , 1 9}$ \\
14603 & 11,13 & $\mathbf{7 6 , 4 2}$ \\
15518 & 11,41 & $\mathbf{7 3 , 7 4}$ \\
19340 & 13,46 & 69,31
\end{tabular}

Tabelle XII.

Eisenchloridlösung Nr. 4.

\begin{tabular}{|c|c|c}
\hline$H$ & $+100 \Delta$ & $+10^{7} \Omega$ \\
\hline 8930 & $+1,675$ & $+18,71$ \\
13587 & $+2,175$ & $+16,13$ \\
14491 & $+2,306$ & $+15,92$ \\
16230 & $+2,458$ & $+15,07$
\end{tabular}

* Die eingeklammerten Werthe sind in der Tabelle I und V nicht enthalten, dieselben sind aus einer anderen Versuchsreihe mit derselben Lösung entnommen.

3. Eine Berechnung des "molecularen Drehungsvermögens" des Eisenchlorids in dem Verdet'schen Sinne ist nicht möglich; übrigens hat bereits $B e c q u e r e{ }^{1}$ ) aus verschieden concentrirten Lösungen verschiedene Werthe für diese Grösse erhalten.

\section{Anhang.}

Es ist oben angegeben, dass, wenn auch das Feld zwischen den durchbohrten conischen Polen nicht auf grössere

1) Becquerel, Wied. Galv. 3. p. 994.

Ann. d. Phgr. u. Chem. N. F. Xxvin. 
Ausdehnung homogen ist, doch der kleine Raum zwischen denselben für die Beobachtungen als hinreichend homogen zu betrachten ist. Es zeigte sich, dass kleine Aenderungen in der Stellung des Glases oder Wassers zwischen den Polen den Betrag der Drehung in diesen Substanzen nicht beeinflussten. Es wurde übrigens, wie oben bereits bemerkt ist, dafür gesorgt, dass die Glasplatte und die Gefässchen mit Wasser und Eisenchlorid stets möglichst genau an derselben Stelle des Feldes sich befanden. Entfernt man aber die conischen Pole weiter voneinander, so wird das Feld zwischen denselben in stärkerem Maasse ungleichförmig, und zwar ist die Intensität desselben nahe an den conischen Eisenspitzen grösser, als in der Mitte. $\mathrm{Da}$ nun das Verhältniss der Drehungen im Eisenchlorid und Wasser oder Glas von der Intensität des Feldes abhängt, so muss dieses Verhältniss sich verschieden ergeben, wenn man bei grösserer Entfernung der Pole die zu untersuchenden Substanzen das eine mal nahe an einen der Pole, das andere mal in die Mitte zwischen beide Pole bringt. Gresagte.

Die folgenden Tabellen XIII und XIV bestätigen das

Die Versuche in Tabelle XIII sind mit dem auch für die oben angegebenen Versuche benutzten Magnet angestellt, die Versuche der Tabelle XIV mit einem Electromagnet der bekannten Ruhmkorff'schen Construction, bei welchem die Entfernung der conischen Polstücke $15 \mathrm{~mm}$ betrug. $E$ und $G$ sollen die Drehungen in der Lösung und im Glas, die Indices $P$ und $M$ die Stellung der Substanz unmittelbar am Pol oder in der Mitte des Feldes bezeichnen.

\section{Tabelle XIII.}

Dicke der Eisenchloridlösung $3,638 \mathrm{~mm}$, des Glases $3,692 \mathrm{~mm}$, Grosser Electromagnet (Poldistanz $10 \mathrm{~mm}$ ), rothes Licht.

\begin{tabular}{|c|c|c|c|c|c|c|c|}
\hline Stromstärke & $G_{P}$ & $-E_{p}$ & $-R_{e g P}$ & $G_{M}$ & & $E_{M}$ & $-R_{e g M}$ \\
\hline 4,9 Ampère & $\begin{array}{l}1^{0} 11,0^{\prime} \\
1 \quad 11,2\end{array}$ & $\begin{array}{l}5^{\circ} 34,1^{\prime} \\
5 \quad 34,4\end{array}$ & $\begin{array}{l}4,705 \\
4,696\end{array}$ & $\begin{array}{ll}1^{0} & 15,8^{\prime} \\
1 & 15,1\end{array}$ & $\begin{array}{l}6^{0} \\
6\end{array}$ & $\begin{array}{l}3,5^{\prime} \\
4,4\end{array}$ & $\begin{array}{l}4,795 \\
4,852\end{array}$ \\
\hline Mittel & $1^{\circ} 11,1^{\prime}$ & $5^{0} 34,25^{\prime}$ & 4,701 & $1^{0} 15,45^{\circ}$ & $6^{0}$ & 3,95 & 4,824 \\
\hline
\end{tabular}




\begin{tabular}{|c|c|c|c|c|c|c|}
\hline Stromstärk $\theta$ & $G_{p}$ & $-E_{P}$ & $-R_{e g p}$ & $G_{M}$ & $-E_{M}$ & $-R_{e g M}$ \\
\hline 4,7 Ampère & $\begin{array}{lr}1^{\circ} & 12,0^{\prime} \\
1 & 10,8 \\
\end{array}$ & $\begin{array}{l}5^{0} 31,7 \\
5 \quad 30,2 \\
\end{array}$ & $\begin{array}{l}4,607 \\
4,660 \\
\end{array}$ & $\begin{array}{l}1^{0} 18,0^{\prime} \\
1 \quad 17,6 \\
\end{array}$ & $\begin{array}{lr}6^{0} & 9,8^{\prime} \\
6 & 10,6 \\
\end{array}$ & $\begin{array}{l}4,741 \\
4,776 \\
\end{array}$ \\
\hline Mittel & $1^{0} 11,4^{t}$ & $5^{\circ} 30,95^{\prime} !$ & 4,634 & $1^{0} 17,8^{\prime}$ & $6^{\circ} 10,2^{\prime}$ & 4,764 \\
\hline
\end{tabular}

Gefäss und Glasdicke $2,251 \mathrm{~mm}$.

\begin{tabular}{|c|c|c|c|c|c|c|}
\hline 5,4 Ampère & $\begin{array}{l}41,4^{\prime} \\
41,4 \\
41,4\end{array}$ & $\begin{array}{ll}2^{\prime \prime} & 41,2^{\prime} \\
2 & 41,8 \\
2 & 40,9 \\
\end{array}$ & $\begin{array}{r}-3,893 \\
-3,908 \\
-3,886 \\
\end{array}$ & $\begin{array}{l}46,6^{\prime} \\
45,9 \\
45,2 \\
\end{array}$ & $\begin{array}{lr}3^{0} & 14,3^{\prime} \\
3 & 15,2 \\
3 & 15,5 \\
\end{array}$ & $\begin{array}{l}4,169 \\
4,252 \\
4,325 \\
\end{array}$ \\
\hline Mittel & $41,4^{\prime}$ & $2^{0} 41,3^{\prime}$ & $-9,898$ & $45,9^{\prime}$ & $3^{\circ} 15,0^{\prime}$ & 4,249 \\
\hline
\end{tabular}

Tabelle XIV.

Dicke der Schicht Eisenchloridlösung und des Glases 2,251.

Ruhmkorff'scher Electromagnet (Poldistanz $15 \mathrm{~mm}$ ). Rothes Licht.

\begin{tabular}{|c|c|c|c|c|c|c|}
\hline Stromstärke & $G_{P}$ & $-E_{P}$ & $-\boldsymbol{R}_{e g} P$ & $G_{M}$ & $-E_{M}$ & $-R_{e g M}$ \\
\hline 13,8 Ampère & $\begin{array}{l}44,9^{\prime} \\
44,6\end{array}$ & $\begin{array}{l}2^{0} 36,5^{\prime} \\
234,8\end{array}$ & $\begin{array}{l}3,485 \\
3,466\end{array}$ & $\begin{array}{l}46,5 \\
49,0 \\
47,2\end{array}$ & $\begin{array}{lr}2^{0} & 59,6^{\prime} \\
3 & 7,0 \\
3 & 3,2\end{array}$ & $\begin{array}{l}3,862 \\
3,816 \\
3,850\end{array}$ \\
\hline Mittel & $44,75^{\prime}$ & $2^{\circ} 35,6^{\prime}$ & 3,476 & 47,85 & $3^{0} \quad 3,3^{\prime}$ & 3,850 \\
\hline
\end{tabular}

Phys. Inst. d. Univ. Strassburg i. E., 1885.

\section{Experimentaluntersuchung uber die Fortfuh- rung der Wärme durch den galvanischen Strom; von H. Haga.}

(Aus den Annales de l'école Polytechnique de Delft für die Annalen bearbeitet vom Verf.).

(Herzu Taf. III Fig. 1-4.)

Die Anwendung der mechanischen Wärmetheorie auf thermoelectrische Erscheinungen hat das Verhältniss klargelegt zwischen den von Seebeck und den von Peltier entdeckten Wirkungen, der durch eine Temperaturdifferenz zwischen den Löthstellen in einer Kette von zwei Metallen verursachten electromotorischen Kraft und den von der Richtung eines Stromes abhängigen in diesen Löthstellen verursachten Wärmeerscheinungen.

Clausius und Thomson, die aus dem Carnot'schen Princip den zweiten Hauptsatz der mechanischen Wärme- 

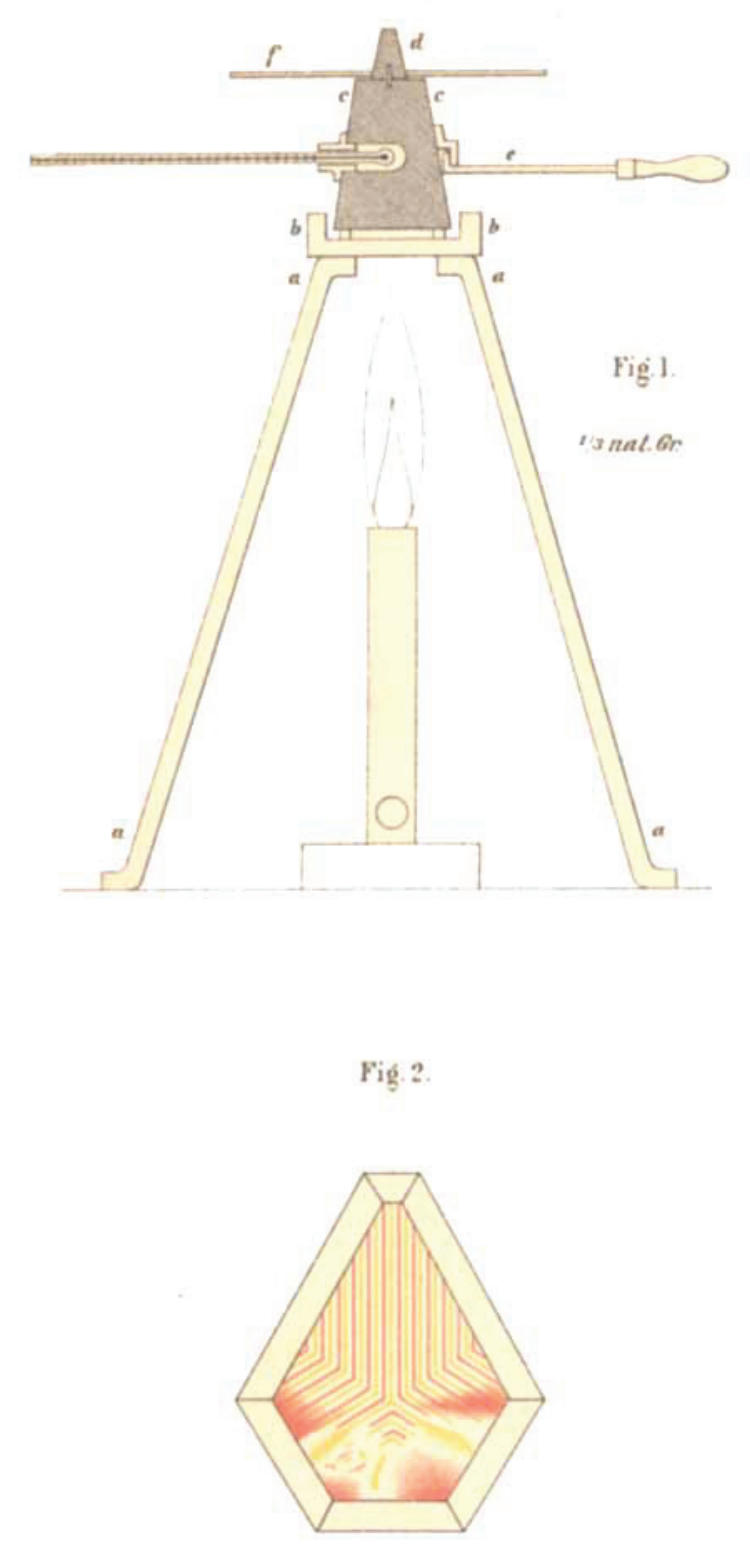
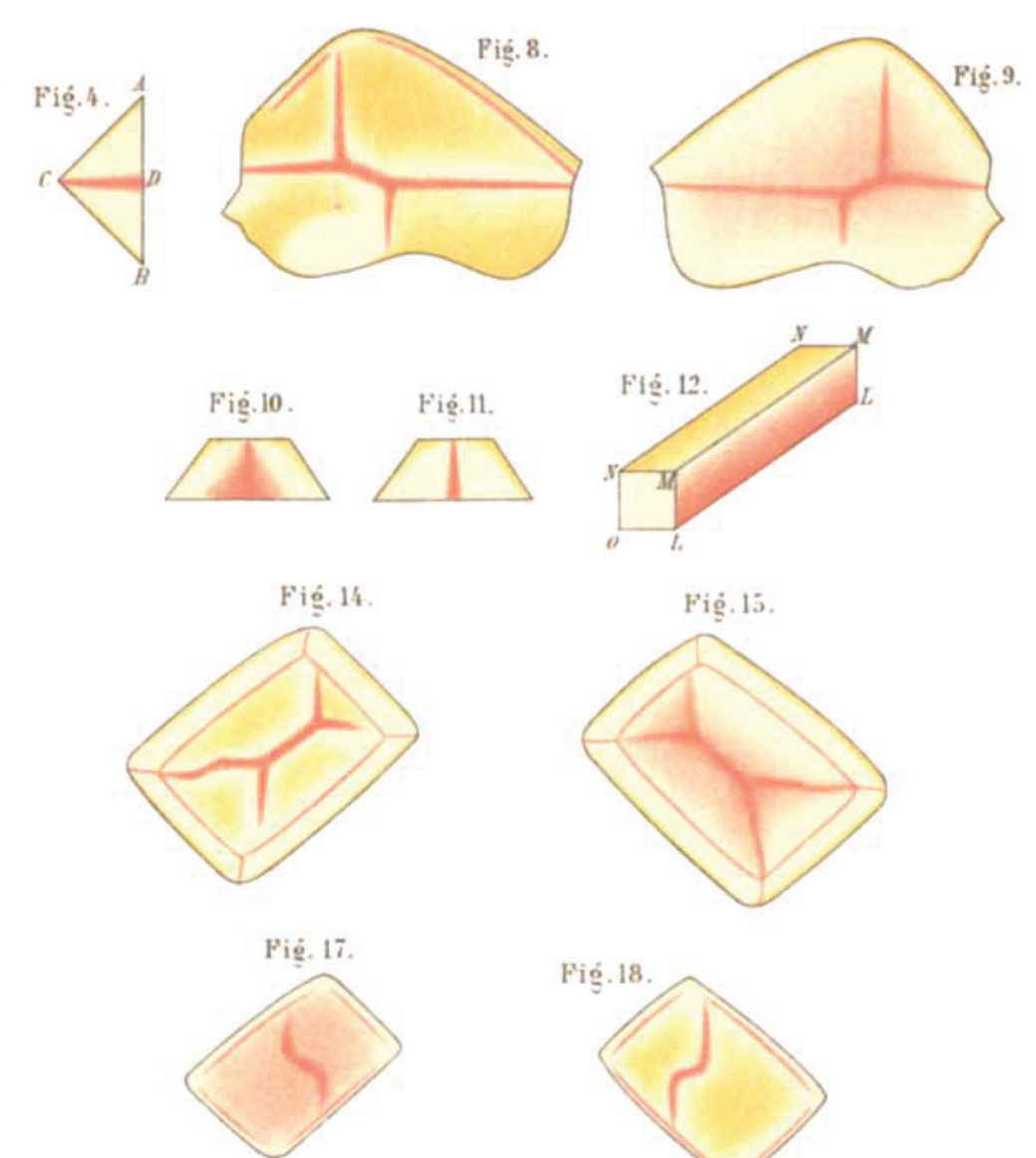

Fịg 18
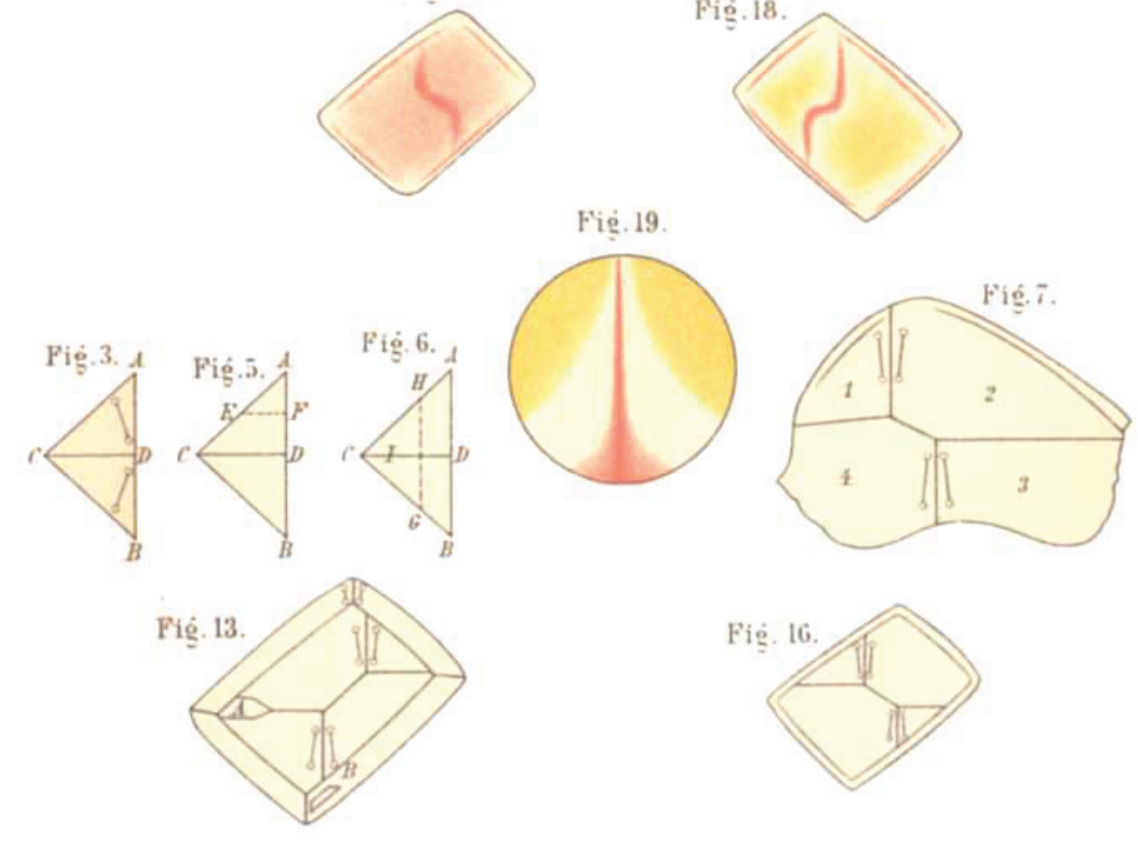

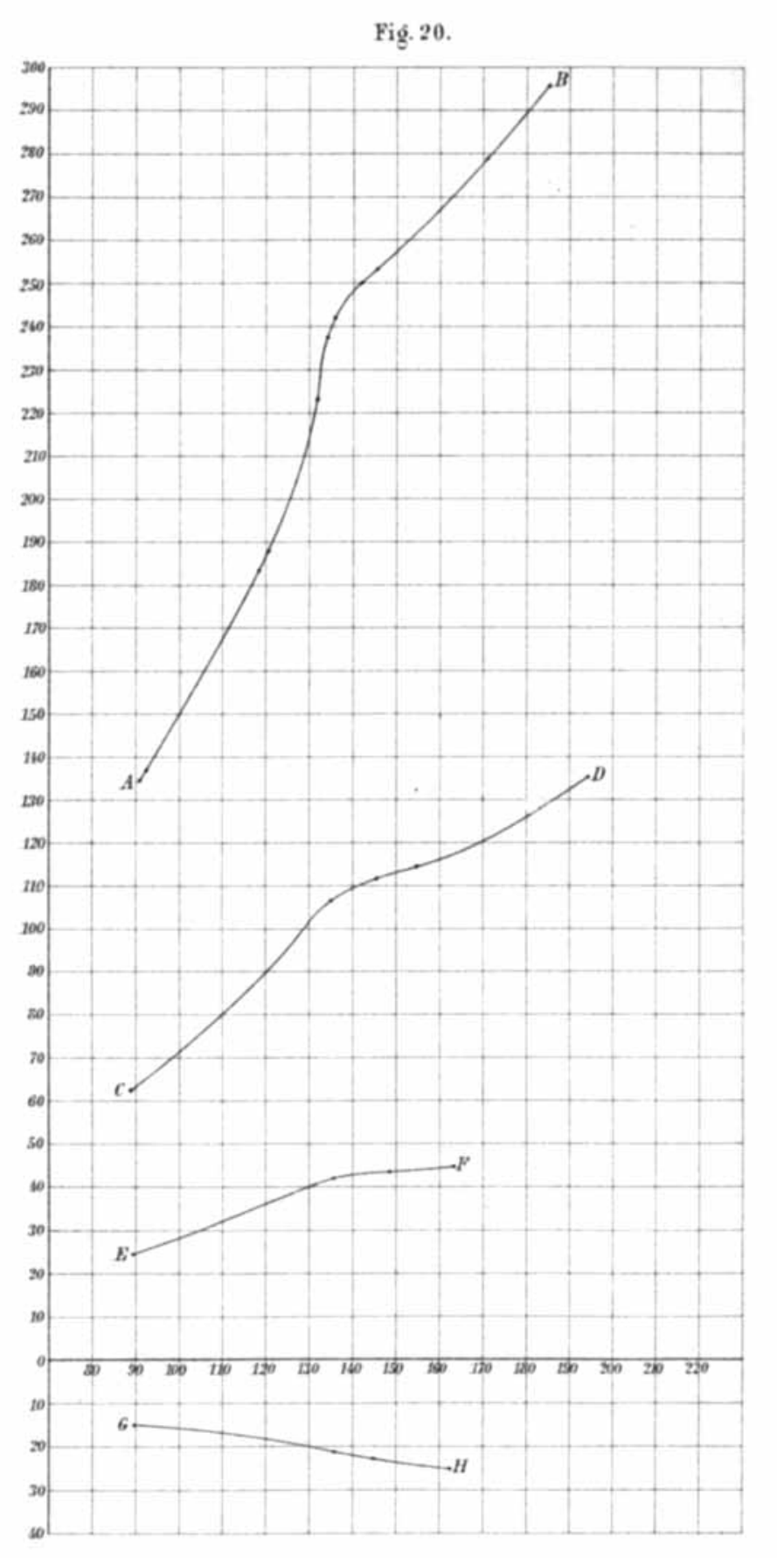

